Kollege Nicol einen solchen über die pathologische Anatomie und Stadieneinteilung der Phthise. Aber auch die folgenden Jahre haben eine immer weiterschreitende Vertiefung und Vervollständigung der Forschungen gebracht und so ist unser aller Wunsch vollauf berechtigt und verständlich, nochmal im großen Rahmen die Frage zu behandeln, Wir glaubten in erster Linie keine besseren Fachkenner hierzu einladen zu können, als wiederum die Kollegen Ranke und Nicol. Im übrigen schien es uns notwendig, daß auch der Standpunkt der praktischen Heilstättenärzte ausgiebig zu Worte kommt; die Kollegen Ulrici, Ziegler und Harms, die auf diesem Gebiete vielfach gearbeitet haben, werden uns daher weitere Berichte geben.

Prof. Dr. Karl Ernst Ranke-München:

\title{
Die Beteiligung der Lunge an den allergischen Stadien der Tuber- kulose.
}

Bei der Beurteilung einer Infektionskrankheit sind stets zwei Reihen von Erscheinungen zu berüeksichtigen, die erst in ihrem Zusammenspiel uns das volle Bild der Krankheit entschleiern können.

Die erste dieser Reihen gehört in den Begriffskreis der ,Sedes morbi“. Die Entdeckung, daß Krankheiten einen Sitz im Körper haben, daß dieser Sitz aufgefunden und erkannt werden kann, und daß diese nachweisbaren lokalen Störungen sich für das Verständnis der krankhaften Vorgänge unentbehrlihh erweisen, bedeutet gewiß einen unermeßlichen Fortschritt der Erkenntnis gegenüber der Vorstellung der Krankheit als eines unfaßbaren Dämons, der sich des Körpers bemächtigt habe. Aber wie überall, so steht doch auch neben dieser neuen Erkenntnis die Sphynx ihrer Reichweite, und je mehr sie sich im Detail der Erfahrung bewährte, desto näher rückte die Gefahr, den Geltungsbereich des gefundenen Prinzips zu überspannen, mit Hilfe des Neugefundenen Alles erklären zu wollen.

Je nachdem große Entdeckungen auf dem einen oder dem anderen Gebiet noch frisch und neu sind und dadurch im Vordergrund des allgemeinen Interesses stehen, neigt die wissenschaftliche Psyche der Völker für ihre Erklärungsversuche sich mehr dem einen oder dem anderen Prinzip als der stärksten schwingenden Apperzeptionsgruppe zu. Neuropathologie, Humoralpathologie und auch Cellularpathologie sind Beispiele solcher Utberspannungen.

Um klar zu machen, was ich meine, möchte ich einige charakteristische Stellen aus dem grundlegenden Werk der modernen pathologischen Anatomie, aus Virchows Cellularpathologie, anführen. Er hat den ganzen Körper durchsucht und überall nur einzelne Zellen gefunden, schließlich wendet er sich der Frage zu, wo die Einheit des Gesamtlebens zu suchen sei. Er weist auch die Behauptung zurück, ,daß das Nervensystem die eigentliche Einheit des Körpers darstelle“. Auch in ihm findet er nur einzelne Zellen und das Prinzip der Arbeitsteilung. Für ihn muß demnach der „,Ausgangspunkt für alle weiteren Betrachtungen“", welche „über Leben und Lebenstätigkeit angestellt werden können, der Schluß sein, $\mathrm{da} B$ in allen Teilen eine Zerspaltung in viele kleine Zentren stattfindet und daß nirgends, soweit unsere anatomische Erfahrung reicht, ein einziger Mittelpunkt 
existiert, von dem aus die Tätigkeiten des Körpers in einer erkennbaren Weise geleitet werden“. An einer späteren Stelle dieser Ausführungen zu Beginn der 14. Vorlesung seiner Cellularpathologie sagt er: „Was man eigentlich sucht, ist. die wirkliche Einheit. Macht man sich die Schwierigkeiten klar, die einer solchen Einheit entgegenstehen, so kann es kaum zweifelhaft sein, daß wir durch die Phänomene unseres Ichs immer wieder irregeführt werden in der Deutung der organischen Vorgänge. Wir, die wir uns als etwas Einfaches und Einheitliches fühlen, wir gehen allerdings immer davon aus, daß von diesem selben Einheitlichen alles bestimmt werden müßte." „Alle heutige Pflanzenphysiologie beruht auf der Erforschung der Zellentätigkeit, und wenn man sich immer noch sträubt dasselbe Prinzip auch in die tierische Ökonomie einzuführen, so ist, wie ich glaube, gar keine andere Schwierigkeit da als die, daß man die ästhetischen und moralischen Bedenken nicht zu überwinden vermag."

Das Ich mit seiner Vorstellung der Einheit führt uns also ,irre"! Wenn schon der Entdecker so sprach, der sich doch der vollen Schwierigkeit der Frage noch bewußt sein mußte, so wird es verständlich, daß seine skrupelloseren Schüler den Rückblick auf die Einheit noch weiter in den Hintergrund stellten, und damit war die "UUberspannung" eingetreten. So wichtig und notwendig es war, das überall im Lebendigen aufzufindende Prinzip der Arbeitsteilung ganz klar herauszuarbeiten, so hätte darüber doch nicht vergessen werden sollen, daß auch ein einheitliches Gesamtleben, das allein erst dieser Arbeitsteilung einen Sinn und die Möglichkeit des Bestehens gibt, nicht nur vorhanden ist, sondern auch einen sehr greifbaren Sitz hat. Auch die Zelle hat noch lebende Organe, selbst die einfachst gebaute. Und sowie wir in ihr unterteilen wollen, erhalten wir auch hier wieder nur mehr Teile. In dieser Beziehung unterscheidet sich das Zellenleben durchaus nicht prinzipiell von dem Leben eines vielzelligen Organismus. Und so gut Virchow und mit ihm wir alle in der Zelle als Ganzem den Sitz des Zellenlebens sehen, so gut müssen wir den ganzen Menschen als unzertrennliche Einheit, als Individuum, als den Sitz seines Lebens ansprechen.

Dieser in seiner Selbstverständlichkeit lächerlich anmutende Satz ist trotzdem ein Schlüssel für alle biologischen und pathologischen Fragen und muß ebensogut „Ausgangspunkt für alle Betrachtungen sein, die über Leben und Lebenstätigkeit angestellt werden können".

Immer wieder handelt es sich darum, den.Blick von den richtig erkannten Teilvorgängen weg auf das Leben des Ganzen und das Leben als Ganzes zurückzuwenden. Auch wir Lungenärzte haben nicht kranke Lungen, sondern kranke Menschen zu behandeln; eine isolierte Lungenkrankheit gibt es erst, wenn die Lunge herausgeschnitten auf dem Sektionstisch liegt. Im Leben aber ist es ein feines, viel verschlungenes, uns nur in groben und vielleicht recht äußerlichen Zügen bekanntes Wechselspiel von Beziehungen, die nach allen Seiten — und auf jeder der Spuren auch wieder hin und her führen - aus dem sich erst das Gesamtgeschehen einer Krankheit zusammensetzt.

Auch der Sitz ,der Krankheit“ ist also in letzter Instanz der Mensch und nicht eines seiner Organe. Stets gilt es, das Gesamtleben zu erfassen und sich nicht zu voreilig mit einem Teilerkennen zu beruhigen. Nicht nur der lokale Krankheitsherd wirkt auf das Gesamtleben, sondern es führen auch sehr wichtige Einwirkun- 
gen vom Gesamtleben auf den Einzelherd zurück. Solche Rückwirkungen oder Reaktionen zeigen sich gerade für den Ablauf der Infektionskrankheiten von ganz ausschlaggebender Wirkung. Von ihnen sind Ausbildung und Ausbreitung der lokalen Störungen gerade bei den Infektionskrankheiten aufs deutlichste beeinflußt. Ihre Berücksichtigung wird uns die zweite Reihe von Erscheinungen ergeben, die wir heute für die Erklärung des krankhaften Geschehens im Gefolge der sog. Infektionen heranziehen können.

\section{Ausbildung der lokalen Krankheitsherde.}

Neben dem Leben der Teile müssen wir also stets auch die lebendige Einheit im Auge behalten. Haben wir einen Tuberkulösen vor uns, so wird unsere nächste Aufgabe gewiß die Auffindung der Sedes morbi dieses Krankheitsfalles sein müssen. Wir werden uns aber z. B. schon von allem Anfang an dessen bewußt bleiben, daB auch die Frage nach dem Krankheitssitz im alten Sinn erst erledigt ist, wenn wir sämtliche lokalen Störungen im gesamten Organismus aufgefunden haben, soweit sie unseren heutigen Untersuchungsmethoden irgendwie zugänglich gemacht werden können. Wie bei jeder Infektionskrankheit, müssen wir weitor zwischen der Verbreitung der Krankheitserreger und der Ausbildung sichtbarer Lokalherde von krankhaftem Strukturbild im Körper unter ihrem Einfluß unterscheiden. Bei der Tuberkulose bildet sich allem Anschein nach am Orte des Eindringens oder in seiner Nähe ein wenn auch kleiner erster Krankheitsherd, der Primärinfekt, bei gleichzeitiger oder rasch aufeinander folgender Ansteckung auch mehrere derartige von außen in den Körper hineingelangte Erstlingsherde. Von ihnen breitet sich dann die Krankheit weiter im Körper aus. Behalten wir zunäehst die Ausbildung der sichtbaren Krankheitsherde im Auge, so können wir für sie vier Ausbreitungsweisen unterscheiden, die etwas verschiedene Bedingungen ihres Auftretens aufweisen und deshalb da, wo sie vorhanden sind, einige Rückschlüsse erlauben.

Die erste derartige Ausbreitungsweise ist das Kontaktwachstum des Herdes, durch das der Herd aus sich heraus durch die homologe Infektion von Zelle zu Zelle in den Randschichten gegen das Gesunde hin sich vergrößert.

Diesem unmittelbaren Herdwachstum stehen drei Arten der Metastasierung, d.h. also der Verschleppung über mehr oder weniger weite, gesund bleibende Zwischenstrecken hin, gegenüber. Sie können nur auf den vorhandenen, vorgebildeten Wegen entstehen und wir finden deshalb solche Verschleppungen in erster Linie innerhalb des Gefäßsystems und zwar sowohl auf dem Wege der Lymphgefäße wie auf dem der Blutgefäße.

Die lymphogene Metastasierung als zweiter Ausbreitungsweg der tuberkulösen Herderkrankung führt zunächst in die regionären Lymphdrüsen, erst nach ihrem Passieren in die Blutbahn.

Zwei Beziehungen sind dabei vor allem im Auge zu behalten. Erstens ist die Lymphdrüse nicht ein Filter, das selbst die kleinsten korpusculären Elemente schon in seiner Randschicht ohne Ausnahme zurückwiese und aus der Lymphe abfiltrierte. Das hat noch Virchow geglaubt und sich dabei auf Befunde von Tusche-, Puiver- und Zinnoberkörnchen nur in den Randschichten unmittelbar beim Vas afferens bezogen. Wir wissen heute, daB ihrer Anlage nach die 
Lymphdrüse ein Klärbecken darstellt. In ihr sind vielgestaltige, maschige Hohlräume in die Lymphgefäße eingeschaltet, in denen die Strömungsgeschwindigkeit sich zweifellos sehr stark verlangsamt, die aber kein absolutes räumliches Hinder. nis für das Passieren auch von viel gröberem korpusculärem Material darstellen. Wir sehen denn auch, daß nicht etwa nur eine vorgelagerte regionäre Drüse erkrankt, sondern regelmäßig eine ganze Drüsenkette. Zum zweiten ist zu berücksichtigen, daß die Lymphgefäße überall in den Saftspalten der Gewebe ihren Anfang nehmen. Dadurch, daß auch der mit der Lymphe wandernde Tuberkelbacillus vom Rande des Herdes, in dem er gewachsen ist, zunächst in die Saftspalten gerät und hier schon sehr bald liegenbleiben, sich vermehren und einen neuen. Kleinen Tuberkel verursachen kann, stehen diese Saftspaltentuberkel in der unmittelbaren Umgebung eines tuberkulösen Herdes auf der Grenze zwischen dem Berührungswachstum und der lymphogenen Verschleppung. Sie werden als Resorptionstuberkel bezeichnet und geraten beim weiteren Wachstum des Mutterherdes in seinen Bereich und werden so zu Bestandteilen jedes größeren „Konglomerattuberkels".

Eine zweite Form der Metastasierung ist die Verschleppung auf dem Blutwege. Haftfläche ist dann eine der vorgelagerten Capillarflächen, also entweder die Lungencapillaren - nach Romberg vor allem die feineren Capillaren der Lungenlymphfollikel - oder die Capillaren des großen Kreislaufes.

Schließlich können Verschleppungen auch auf allen anderen vorhandenen, vorgebildeten Wegen erfolgen. also in Drüsengängen, den großen Lymphräumen, den Sehnenscheiden z. B., dann im Darmrohr, im Bronchialbaum, im Genitaltrakt usw. Für diese dritte Metastasierungsform habe ich die Bezeichnung intracanaliculäre Metastasierung vorgeschlagen und leider auch noch keine bessere kurze Benennung dafür auffinden können. Sie faßt also alle Metastasierungen in vorgebildeten Höhlen- und Röhrensystemen des Körpers zusammen, soweit sie nicht den Blut. und Lymphgefäßsystemen angehören.

Der Kern meiner Beobachtungen ist nun darin enthalten, daß diese verschiedenen Ausbreitungsweisen sich in den verschiedenen Entwicklungsphasen der Krankheit ganz verschieden zu einem momentanen Gesamtbild der Krankheit zu kombinieren pflegen, so daß auf diese Weise sich verschiedene - d. h. biologisch ungleichwertige--Krankheitsphasen unterscheiden lassen. Die Tuberkulose als typische chronische und rekurrierende Krankheit zeigt diese verschiedenen Phasen oft zeitlich ungeheuer weit auseinandergezogen. Nur die von vornherein sehr rasch verlaufenden schweren Erkrankungen zeigen einigermaßen den gedrängten Ablauf, den wir von den akuten Infektionskrankheiten her kennen. Die weit überwiegende Mehrzahl aller Infektionen schreitet so langsam fort, daß wir oft Jahre und selbst Jahrzehnte lang das gleiche Entwicklungsstadium der Krankheit bestehen bleiben sehen und daß bei den langsamsten, leichtesten Formen selbst das ganze Leben zu einer vollen Entwicklung bis zu den Endstadien nicht mehr ausreicht. Zeiten des scheinbaren Stillstandes wechseln dabei mit Zeiten des rascheren Fortschreitens. Klinische Heilung ist bei den leichten Fällen ungemein häufig, während eine völlige pathologisch-anatomisch nachweisbare Ausheilung selbst bei wenigen kleinen Krankheitsherden zu den Seltenheiten gehört.

Bleiben wir zunächst immer noch bei der alleinigen Betrachtung der Krank- 
heitsherde nach Zahl, Größe und Anordnung, so lassen sich drei aufeinanderfolgende Hauptanordnungen unterscheiden.

Die erste ist diejenige des Primärkomplexes. Er setzt sich zusammen aus dem Ort der ersten Ansiedlung - dem eigentlichen Primärinfekt -, und den lymphogenen Metastasen in den ihm regionären Lymphdrüsen und Lymphgefäßen. Offenbar haben die Bacillen außer ihrem allerersten Ansiedlungsplatze in den unmittelbar anschließenden Lymphgefäßen und den nächsten vorgelagerten Lymphdrüsen zunächst die besten Ansiedlungsbedingungen. Wir werden dabei zum Verständnis der so auffallend rasch sich bildenden Drüsenmetastasen daran denken dürfen, daß die Bacillen in den regionären Lymphdrüsen geradezu angesammelt, wenn auch nicht restlos abfiltriert werden. Die zur Herdbildung nötige Konzentration und Ruhe wird also hier ziemlich gleichzeitig mit dem primären Herd erreicht.

Bei der großen Anzahl leichter Infektionen hat dieser Primärkomplex einen beträchtlichen zeitlichen Vorsprung vor den übrigen Ausbreitungsweisen und kann deshalb in dieser Zeit als ,,isolierter Primärkomplex" ohne weitere sichtbare Sedes morbi beobachtet werden. Die Krankheit kann in diesem Stadium schon zur Abheilung kommen.

Als Zeichen einer weiter fortgeschrittenen Erkrankung finden wir das Auftreten zweifelloser hämatogener, also auf dem Blutweg verschleppter Metastasen. Die Krankheit kann sich damit im ganzen Körper verbreiten, obwohl immer zu beachten ist, daß die Organe für das Haften hämatogener Metastasen recht verschieden empfänglich sind. Die rasche und reichliche hämatogene Ausbreitung ergibt das bekannte Bild der Miliartuberkulose. Sie kommt aber sowohl akut als subchronisch und chronisch vor, und die von ihr gesetzten Herde können zahllos, oder doch sehr reichlich bis äußerst spärlich sein. Die akute allgemeine Miliartuberkulose ist also nur der extremste Fall einer auch sonst gesetzmäBig sich einstellenden Ausbreitungsweise.

Gegenüber dem geschilderten Primärkomplex kann eine Krankheit, bei der hämatogene Ausbreitungsweise nachgewiesen ist, als sekundäre, gegenüber dem stets auf ein Organ beschränkten Charakter des Primärkomplexes aber bei der Möglichkeit, im gesamten Körper an jeder Stelle aufzutreten, als generalisierte Tuberkulose bezeichnet werden. Spätere Entwicklungsformen unterscheiden sich sehr deutlich von den geschilderten durch das Zurücktreten einzelnerAusbreitungsweisen.

Der Primärkomplex zeigt neben der lymphogenen Ausbreitung ein sehr ausgesprochenes Kontaktwachstum der Herde. Neben den bekannten rundlichen Primärinfekten bilden sich auch zusammenhängende verkäsende Herde in den zugehörigen Lymphdrüsen, die in ihrer Gesamtheit meist größer sind als der oder die Primärherde zusammengenommen. Auch in den Drüsen entstehen also nicht nur einzelne zerstreute Tuberkelehen, sondern zusammenhängende größere Käseherde.

Ist der isolierte Primärkomplex auf Kontaktwachstum und lymphogene Metastase beschränkt, so tritt im sekundären Stadium neben diese beiden Ausbreitungsweisen die hämatogene und früher oder später auch die intracanaliculäre Metastasierung. Der Krankheit sind nunmehr also alle Wege offen und sie bedient sich je nach den örtlichen und sonstigen Bedingungen bald des einen, bald des anderen, bald aller vier gleichzeitig. Sie hat damit ihren Höhepunkt, 
ihre Acme, erreicht. Bei den von vornherein schwer verlaufenden Formen tritt in diesem Stadium früher oder später der Tod ein.

Nur die leichteren, langsamer verlaufenden Erkrankungen können über dieses Stadium hinaus zu weiteren Entwicklungsformen vordringen, soweit sie nicht, was bei der Tuberkulose in jedem Stadium möglich zu sein scheint, klinisch, in seltenen Fällen vielleicht auch anatomisch, ausheilen. Bei diesem weiteren Fortschreiten entwickelt sich allmählich ein neues Bild. Die humoralen Metastasen - die sich ja immer ruckweise aubilden - treten dabei zurück, werden immer seltener, geringfügiger oder vereinzelter, und werden schließlich mehr oder weniger rudimentär oder bleiben ganz aus. Es handelt sich dabei nicht darum, daß den Bacillen in diesen Spätstadien der Weg in die Lymphgefäße und Lymphdrüsen oder in die Blutgefäße vollkommen verlegt wäre. Das läßt sich sogar mit Sicherheit ausschließen. Sondern es handelt sich darum, daß auch bei Verschleppungen von infektiösem Material Metastasen nicht mehr recht angehen, jedenfalls keine größeren, aus sich selbst wachsenden Sedes morbi erzeugen. Daß infektiöses Material noch in die Blutbahn gelangt, wissen wir nicht nur seit Liebermeister direkt durch die Blutuntersuchungen, sondern auch aus dem Nachweis eben der rudimentär bleibenden Herdchen in Lymphdrüsen und anderen Organen, wie z. B. im Auge, und ihrer Häufung in den langen, qualvollen Wochen einer verglimmenden Vita minima, in denen der reine Phthisiker zugrunde zu gehen pflegt. Dieses Ausbleiben der humoralen Metastasen ist also ein sehr wichtiger Zug im Krankheitsbild und wird uns je nach seinem Grade berechtigen, von einer Spätform dè Tuberkulose, also einer spätsekundären oder tertiären Erkrankung zu sprechen.

Schon während des Abheilens der spontan heilenden oder therapeutisch ausreichend günstig beeinflußten sekundären Formen tritt die weitere Ausbreitung durch humorale Metastasierung gewöhnlich stark zurück. Wo sie das nicht tut, tritt eben keine Heilung ein. Wo die humoralen Metastasen aber ausbleiben, entscheidet über das weitere Schicksal des Kranken und der Krankheit das Kontaktwachstum der bis dahin gesetzten Herde und die intracanaliculäre Verschleppung.

Das Schlußbild innerhalb der Entwicklungsphasen der menschlichen Tuberkulose bildet eine Erkrankung des offenbar für Infektion wie für Weiterentwicklung der Krankheit disponiertesten Organes, der Lunge. In diesen echten tertiären Formen, über deren Entstehung wir noch wenig Sicheres wissen, finden wir nur mehr zwei Ausbreitungsweisen: das Kontaktwachstum der vorhandenen Herde und die bronchogene, - also intracanaliculäre - Ausbreitung. Wir finden also am Ende der Krankheitsentwicklung wieder eine Art Analogon des isoliertenPrimärkomplexes, eine isolierte Organtuberkulose der Lunge, diesmal aber nicht zusammengesetzt aus durch Kontaktwachstum und lymphogene Ausbreitung, sondern aus Kontaktwachstum und intracanaliculäre Metastasierung entstandenen Herden.

\section{Ausbildung verschiedener Reaktionsweisen des Befallenen.}

Bei diesem Hervortreten einer bestimmten Organerkrankung und dem Zurücktreten der humoralen Metastasen sehen wir aufs deutlichste zwei Faktoren 
wirksam werden, die uns zu einer zweiten Reihe von Vorgängen überleiten. Es sind das die Disposition und ihr Widerspiel, die Immunität. Beides sind kurze Sammelnamen für ein offenbar sehr verwickeltes Geschehen und bedürfen aufs dringendste einer näheren begrifflichen Verarbeitung. Es ist selbstverständlich, daß diese begriffliche Verarbeitung ihren Wert ausschließlich aus der Vollständigkeit erhält, mit der sie das heute bekannte Tatsachenmaterial zu ordnen und als Leitfaden - als sog. heuristisches Prinzip - für weitere Beobachtungen und Entdeckungen $\mathrm{zu}$ dienen vermag.

Auch bei der Immunität und bei der Disposition treffen wir sofort wieder auf die Frage nach dem Sitz des Geschehens, denn wir stoBen bei ihrer Erforschung sofort auf typische Äußerungen des Gesamtlebens, des Lebendigen als Einheit, und neben diesem wieder anf Vorgänge, die sich in einzelnen Teilen des lebendigen Körpers lokalisieren. Es ist sehr reizvoll, der Geschichte unserer wissenschaftlichen Begriffe nachzugehen. Sie zeigen eine Art Ontogenese mit Analogien zum biogenetischen Grundgesetz. So wiederholt denn auch die Begriffsbildung z. B. bei der Immunität Entwicklungsphasen, die der Lebens- und mit ihm der Krankheitsbegriff als Ganzes schon durchschritten haben. Das crste Stadium enthält noch den vollen Blick auf die Einheit, die weitere Analyse erst gibt dem vereinfachenden allgemeinen Begriff nach und nach die Einzelheiten eines schließlich überreich werdenden Beobachtungsmaterials. Damit wird die Einheit wieder fraglich. Der antizipierte Begriff muß sich vor dem Beobachtungsmaterial schließlich noch einmal legitimieren und wird verworfen oder aufgeteilt werden müssen, wenn er sich an ihm nicht bewähren konnte.

Solange wir von der Immunität nur die gröbsten Unarisse kannten, lag es nahe, sie unter allen Umständen als Änderung des Gesamtlebens aufzufassen und zwar als allgemein und typisch geänderte Reizantwort auf eine gleichbleibende Reizursache. So wie man aber näher zusieht, genauer untersucht, und über ein allmählich anwachsendes Beobachtungsmaterial verfügt, findet man, daß sich neben Gesamtänderungen der Reizantwort auch analoge Teilvorgänge an einzelnen Organen, Geweben, Zellen auffinden lassen.

Disposition und Immunität gehören als begriffliches Gegenpaar so notwendig zueinander wie Licht und Schatten. Wenn wir also unter Disposition, wie allgemein üblich, ein über das Übliche hinausreichendes Erleichtertsein des Erkrankens verstehen wollen, so ergibt sich daraus schon die Berechtigung unter Immunität die deutlich nachweisbaren Behinderungen des Erkrankens zusammenzufassen. Von der Disposition wissen wir, daß sie offenbar von sehr verschiedenen Faktoren beeinflußt und hervorgerufen werden kann. Wir kennen aber auch schon Immunitäten von prinzipiell ganz verschiedenem Mechanismus oder Chemismus und von ganz verschiedenem Grade und Dauer. Wie wir schlieBlich Organdispositionen oder Systemdispositionen kennen, so treffen wir in dem von mir zusammengetragenen Beobachtungsmaterial auf entsprechende Teilimmunitäten. Sie sind bei den beschriebenen Tuberkuloseformen ganz augenfällig. Sie müssen näher studiert und in unsere Gedankenrechnung eingesctzt werden. Es wird vergebliche Mühe sein, wenn man versucht, sie wegzudisputieren.

Als Definition von Disposition ergibt sich nun das Eintreten von Krankheit oder Krankheitserscheinungen ganzer Organismen oder ihrer Teile unter Bedingungen, 
unter denen gleichartige Individuen oder ihre Organe sonst nicht in dieser Art erkranken würden, und dementsprechend für Immunität das Ausbleiben von Krankheit oder Krankheitserscheinungen unter Bedingungen, unter denen sie sonst eingetreten wären. Der springende Punkt für die Beurteilung ist dabei, daß der Grund für das Auftreten und Ausbleiben von Krankheit bei der Gleichheit aller übrigen Bedingungen nur mehr in Eigenschaften des Lebendigen selbst gesucht werden kann. Unter spezifischer erworbener Immunität ist dann das Ausbleiben von Krankheit oder einzelnen Krankheitserscheinungen infolge des Überstehens oder Vorhandenseins der gleichen Krankheit oder der künstlichen Vorbehandlung mit dem gleichen Krankheitserreger oder von ihm stammender Produkte zu verstehen.

Es kann. keinem Zweifel unterliegen, daß das Ausbleiben der humoralen Metastasen in der geschilderten Weise als eine Teilimmunität aufgefaßt werden muß, und daß sie tatsächlich häufig und leicht beobachtet werden kann. Da es sich um nichts prinzipiell Neues handelt, ist es wissenschaftlich ratsamer, mit dem alten Begriff auch das alte Wort zu verwenden als einen neuen terminus technicus mit allen seinen Gefahren der Unverständlichkeit und der Verwechslung einzuführen. Wir müssen also den Begriff der Immunität unterteilen an der Hand der tatsächlichen Beobachtungen. Wie wir schon langdauernde und kurzdauernde Immunitäten, sowie absolute und relative Grade derselben kennengelernt haben, so stoßen wir also hier auf eine Teilimmunität, die zwar das Krankheitsbild sehr wesentlich zu verändern vermag, nicht aber notwendig die dauernde Heilung der Gesamterkrankung und völlige Verhinderung jeder Neuansteckung herbeiführen muß. Es ist übrigens ja auch schon seit Behring von einer ,humoralen " und einer ,cellulären" Immunität gesprochen worden, Unterscheidungen, die offenbar nach einer ähnlichen Richtung deuten, wenn sie auch mehr theoretische Konzeptionen als der Ausdruck für tatsächliche Beobachtungen waren.

Die Untersuchung der lokalen und allgemeinen Reaktionsweisen scheint mir nun ein weiteres, für die schärfere Erfassung der Entwicklungsphasen der Tuberkulose wichtiges Material zu liefern, und zwar glaube ich in ihnen den speziellen Ausdruck eines allgemeinen Gesetzes für den Ablauf der Infektionskrankheiten wieder erkennen zu können.

Auch dieses Gesetz der Infektionskrankheiten ist wieder in noch allgemeineren Eigenschaften des Lebendigen verankert. Wir wissen alle, daß die gleiche reizauslösende Ursache vom gleichen Geschöpf durchaus nicht stets wieder auf die gleiche Weise beantwortet wird. Es ist sogar sehr viel häufiger, daß spätere Antworten anders ausfallen als die erste. Die Antwort kann sich in ihrem Wesen gleich bleiben und nur in ihrem Grade verändern; die Antwort kann dann rascher, geordneter und kräftiger ausfallen, oder sie kann langsamer und geringer werden, bis sie ganz ausbleibt. Wir sprechen in dem einen Fall von Utbung, in dem anderen Fall von Gewöhnung. Bei den den allgemeinen Lebensablauf störenden Reizursachen, unter denen wir die krankheitsauslösenden Wirkungen der Außenwelt zu suchen haben, können wir zwei ähnliche, ganz große, erste Unterteilungen vornehmen. Es gibt Reize, die bei ihrer Wiederholung immer fühlbarer, schließlich vollkommen unerträglich werden, deren Wiederholung also die Reizbarkeit steigert und solche, die nach und nach immer weniger, schließlich überhaupt nicht mehr gefühlt werden. Die erste Reihe der erzielten Reizantworten können 
wir als Überempfindlichkeit, die zweite als Immunität bezeichnen. Wir gewinnen damit den Anschluß an ein sehr vielfältiges und heute noch sehr verworrenes Beobachtungsmaterial, das unter den Schlagwörtern Anaphylaxie, Überempfindlichkeit und Immunität gesammelt ist, während beide Erscheinungen nach Pirquet unter dem allgemeinen Begriff der Reizantwortsänderungen, der Allergie, zusammengefaBt werden.

Man hat diese Reizbarkeitsänderungen zunächst in experimentellen Versuchen mit den Erregern oder ihren Leibes- und Ausscheidungsstoffen studiert. Es ist aber schon von Pirquet, dem wir die wichtigsten Beobachtungen auf diesem Gebiet verdanken, gezeigt worden, da $B$ sich die von ihm entdeckten Änderungen auch im Krankheitsablauf selbst ohne weiteres erkennen lassen. Die gleiche Betrachtungsweise habe ich auch auf das anatomische und mikroskopische Strukturbild des Krankheitsherdes angewendet, und sie hat sich auch hier für das Verständnis des krankhaften Geschehens als verwendbar erwiesen.

\section{Gesetz des cyklischen Ablaus der Infektionskrankheiten.}

Bleiben wir wieder bei den großen Außenlinien der uns bis heute zugänglich gewordenen Krankheitsbilder, so sehen wir, daß jede Infektionskrankheit naturgemäß von einer gewissen normalen jungfräulichen Erregbarkeit, einem Zustand der Unberührtheit, ihren Ausgang nimmt. Unter der dauernden Einwirkung des infektiösen Virus stellt sich, nach einer gewissen Vorbereitungszeit - dem Inkubationsstadium - und einer Periode der ersten Ansiedlung, zunächst eine deutliche Reizbarkeitssteigerung ein, die sich durch Zunahme oder auch Neueintreten sog. entzündlicher Erscheinungen zu erkennen gibt. Diese entzündlichen Erscheinungen sind im wesentlichen akut und exsudativ. Sie sind uns allen bekannt aus den am stärksten ins Auge fallenden Beispielen akuter Allgemeininfektionen, z. B. also den Exanthemen bei Scharlach, Masern, Pocken usw. Es ist nicht notwendig, daß diese entzündlichen Erscheinungen sich ausschließlich oder vorwiegend auf den äußeren Bedeckungen zeigen, sie sind hier nur am auffälligsten. Mit der Weiterentwicklung der Erkrankung steigern sich die Störungen des Lebensbetriebes durch die Krankheitserscheinungen. Die Ausbreitung der Krankheitserreger, die Ausbildung zahlreicher Krankheitsherde mit lokalen Störungen unmittelbar lebenswichtiger Organe oder die Schwere der Allgemeinerkrankung kann in dieser Entwicklungszeit zum Tode führen. Ubersteht der Organismus diesen Angriff - wobei dahingestellt sein soll, ob durch die Steigerung der Reizantwort oder trotz derselben - so machen sich bald Erscheinungen einer Reizminderung, bei den akuten Infektionskrankheiten meist einer rasch einsetzenden und sehr vollständigen Gesamtimmunität geltend. Unter ihnen kann - bei Fehlen nicht rasch genug reparabler Organstörungen, die sonst als Sedes morbi von verschiedenem Charakter und sehr verschiedener Gefährlichkeit bestehen bleiben und den weiteren Krankheitsablauf beherrschen - die Rekonvaleszenz und Heilung eintreten.

Dieses „Gesetz des cyclischen Ablaufs" der alten Autoren ist so typisch, daß es schon lange vor der Entdeckung der Infektionserreger zur richtigen Charakterisierung der durch ein damals nur supponiertes Contagium vivum ausgelösten Krankheitsformen dienen konnte. Es läßt sich auch bei der Syphilis und bei der 
Tuberkulose wieder erkennen. Auch bei der Tuberkulose folgt auf ein erstes Stadium mehr oder weniger rasch eine eigenartige Reizbarkeitssteigerung, durch die akut entzündliche Erscheinungen einen sehr ins Auge fallenden Anteil am Krankheitsbild erhalten. In dieser Entwicklungszeit, die den frühen Zeiten der hämatogenen Dissemination entspricht, wenigstens soweit erkennbare krankhafte Wirkungen von ihr ausgehen, finden wir nicht nur sehr aufällige, spontane, entzündliche Erscheinungen auf der Haut, den Schleimhäuten und in den Randpartien der Sedes morbi, sondern wir können die gleiche Reaktionsänderung auch im Experiment nachweisen. Bei der Einverleibung von ganzen Bacillen, Bacillenleibesstoffen oder Tuberkulin sehen wir diese Reaktionsweise in akuten exsudativen Entzündungen am Applikationsort und in der Randzone von lokalen Herden auftreten. Da das Tuberkulin beim gesunden, von Tuberkulose unberührten, nicht umgestimmten Organismus derartige Wirkungen nicht besitzt, tritt diese Beobachtung in Analogie zur echten Anaphylaxie, also z. B. dem Nachweis der Sensibilisierung gegen ein bestimmtes Eiweiß mit Hilfe der Stichreaktion.

Da diese Umstimmung sich an allen Körperstellen nachweisen läßt, so können wir nicht umhin, sie auf die parenterale Verarbeitung von Bacillen und deren Derivaten zu beziehen. Bleiben wir dabei im üblichen naiv-stofflichen Bild der heutigen Humoralpathologie, so werden wir an Bildung von Reaktionsstoffen und ihre sehr weitgehende Verbreitung im Körper denken.

Die Betrachtung der Reaktionsweise führt uns also hier von den Sedes morbi selbst weg, denn es ist unwahrscheinlich, daß diese Verarbeitung ausschließlich oder auch nur vorwiegend in den lokalen Krankheitsherden sich vollzieht. Seit den Untersuchungen von Liebermeisler wissen wir, daß infektiöses Material in großem Umfang in die Blutbahn gerät, ohne daß eine entsprechende Anzahl von lokalen Herden sich ausbildet; und es erscheint heute als gut begründete Ansicht, daß gerade dieses scheinbar wirkungslose Material zum guten Teil abgebaut und damit zur Ursache der Reaktionsänderung wird. Im Beginn der sekundären Phase begegnen wir also neben der hämatogenen Ausbreitung der Lokalherde auch einer Sensibilisierung des Gesamtorganismus, bei der ebenfalls der Blutweg eine ausschlaggebende Rolle spielt. Diese Sensibilisierung ist zunächst wie bei allen bisher durchgeprüften Eiweißarten in gewissem Sinne unspezifisch, insofern als ihr Produkt bei allen einen sehr gleichartigen allgemeinentzündlichen Charakter trägt.

\section{Sekundäre Tuberkulosen.}

$\mathrm{Zu}$ diesen vorwiegend entzündlichen Manifestationen der Tuberkulose gehören z. B. die meisten Formen der Tuberkulide und ganz besonders typisch die Phlyctäne. Sie stellen örtliche Störungen dar, die trotzdem keine dauernden Sedes morbi werden. Ähnliche entzündliche Reaktionen stellen sich aber auch für mehr oder weniger lange Dauer in den Herden und namentlich in ihren Randzonen ein. Dabei kann der in dieser Form reagierende Herd selbst sehr gering. fügig sein. Wir wissen für die Tuberkulide und Phlyctänen, daß hier offenbar schon geringfügigste Mengen eines mehr oder weniger stark abgebauten Virus ohne jede Vermehrungsfähigkeit am Orte ihres Haftenbleibens solche Reaktionen auslösen können; wir dürfen uns also nicht wundern, wenn in dieser Entwicklungs- 
zeit der Krankheit auch geringfügige Herde in den Drüsen oder auch in anderen Organen, z. B. in den Lungen zeitweise sehr starke entzündliche Reaktionen verursachen. So treffen wir z. B. für unser spezielles Beobachtungsgebiet auf hartnäckige Erkrankungen im wesentliohen katarrhalischer Natur, als deren Grundlage nur vergleichsweise recht geringfügige Herde und Herdohen in Lungendrüsen und im Lungenbindegewebe sich nachweisen lassen. Diese Erkrankungen wurden vor meinen Untersuchungen entweder als larvierte Tuberkulasen bezeichnet oder mit der isolierten Phthise, also dem Endstadium der Tuberkulose, zusammengeworfen, immer vorausgesetzt, daß sie überhaupt richtig erkannt wurden. Sie sind aber weder larviert, denn sie lassen sich mit Hilfe des Röntgenbilds und der klinischen Untersuchung ohne weiteres diagnostizieren, noch sind sie, wie später noch ausgeführt werden soll, mit der Phthise zu identifizieren.

Von diesen humoral sich ausbreitenden Tuberkulosen mit großer Neigung zu entzündlicher Randreaktion gibt es alle Schweregrade, von den leichtesten, nur durch einzelne Tuberkulide und Phlyktänen und die positive Tuberkulinhautreaktion sich verratenden Formen, bis zu den schwersten, unter Bildung zahlreicher ausgedehnter Lokalherde und der miliaren Form der Ausbreitung zum Tode führenden Formen. Dazwischen liegt das große Gebiet der chronisohen generalisierenden Tuberkulose mit ihren zahlreichen, zu ausgedehnten entzündlichen Einschmelzungen führenden Herden.

Ausgedehnte Lungenherde sind immer eine äußerst schwere Erkrankung. Während man selbst bei den größten Weichteileinschmelzungen dieser sekundären Tuberkulosen und auch bei sehr großer Zahl derartiger Herde im Gebiet des großen Kreislaufs die Hoffnung durchaus nicht aufzugeben braucht, sondern ans Wunderbare grenzende Heilungen, vor allem unter Sonneneinwirkung, geradezu zur Tagesordnung gehören, so gilt das gleiche nicht mehr für die Lungen. Zwar kommen auch große Hilusdrüsenherde noch zur Abheilung, aber größere einschmelzende Lungengewebsherde doch ungleich seltener als die entsprechenden Herde z. B. des Bindegewebes und der Knochen. Die Lunge ist offenbar ein Organ, das mit der tuberkulösen Lokalerkrankung ganz besonders schwer fertig wird. Immerhin gilt auch noch für die hierher gehörigen Lungenerkrankungen, daß es sich um vergleichsweise akute Entzündungsformen handelt und daß gelegentlich auch in der Lunge hier Heilungen vorkommen, wie man sie später nicht mehr zu sehen bekommt. Das gilt besonders auch von den sekundären kavernösen Lungentuberkulosen der Kinder.

Klinisch ist aber ein anderer Zug für diese Krankheitsbilder von allergrößter Wichtigkeit. Soweit die akute Entzündung nur Reaktion des Organismus ist, ist sie ebenso reparabel wie die Stichreaktion bei irgendeiner der Tuberkulinreaktionen. Wir können deshalb sehr hochgradige exsudative Entzündungen, selbst wo sie durchaus den Charakter katarrhalisch-pneumonischer Erkrankungen annehmen, bei derartigen Tuberkulosen sehr rasch entstehen, aber auch erstaunlich rasch wieder abklingen sehen. Wir finden dann bei einer Person, die vorher vielleicht nur ganz geringfügige, lokalisierte, chronisch bronchitische Erscheinungen in der Nähe der dauernden Sedes morbi aufgewiesen hatte, mit einem Schlag ein sehr schweres klinisches Bild, Dämpfung, viel entzündliches Sekret, Erscheinungen der.Konsonanz von verschiedener Stärke, so daß man geneigt ist, 
an eine schwere Ausbreitung der früher gutartigen und stationären Tuberkulose zu glauben. Es kann tatsächlich dieser Zustand in eine solche Ausbreitung überführen; viel häufiger ist es aber, daß es sich lediglich um eine akute entzündliche Reaktion handelt, die nahezu so rasch wieder verklingt, wie sie entstanden ist. Derartige klinische Bilder, zu denen z. B. ein großer Teil der sog. eintägigen Pneumonien der Kinder zu rechnen ist, - ich habe einen solchen von anderer Seite als eintägige Pneumonie angesprochenen Fall bei kindlicher Hodentuberkulose schon 1894 mitbeobachtet - sind in ihrer völligen Unvereinbarkeit mit dem klinischen Bild der echten isolierten Phthise der eigentliche Anstoß zu meinen Untersuchungen gewesen. Sie müssen jedem Lungenarzt bekannt sein. Allerdings kommen sie in der allgemeinen und namentlich der Kinderpraxis viel häufiger vor als in Sanatorien. Sie sind bei den Tuberkulosen mit Herden im großen Kreislauf viel häufiger als bei den klinisch sich auf die Lungen beschränkenden Formen. So kommt es, da $B$ in den Lungensanatorien gerade diese Formen vergleichsweise sehr selten sind. Ich selbst habe sie bei 6 jähriger Tätigkeit im Sanatorium dort niemals zu Gesicht bekommen, wohl aber häufig bei meiner Tätigkeit im Kinderspital, als Schularzt, als Fürsorgearzt und in der Stadtpraxis.

Die eigenartige spezifische erworbene Überempfindlichkeit dieser frühen Tuberkuloseformen zeigt eine ganze Reihe der interessantesten Beziehungen. Ich will mich dabei durchaus auf die Beobachtungen beschränken, um so mehr als Versuche zu einer theoretischen Deutung heute noch nur zu sehr problematischen Ansichten führen können. Die Beobachtung am Kranken zeigt, daß sie sich häufig vergesellschaftet mit einer Art Gruppenüberempfindlichkeit. Was dabei das Primäre ist, läßt sich heute noch nicht unterscheiden, doch sehen wir alle möglichen Formen von Ekzem, dann von Asthma, von Pollenüberempfindlichkeit usw. gerade mit Formen der Tuberkulose vergesellschaftet, die in dieser Überempfindlichkeitsperiode ,stecken geblieben" sind. Es sind leichte generalisierte Tuberkulosen, sowohl nach der Art der Anordnung und der Größe der Sedes morbi, wie nach der Reaktionsweise gegen die Bacillenstoffe, die wir ganz außerordentlich häufig neben den genannten Erkrankungen nachweisen können. Die tuberkulöse Erkrankung zeigt dabei meist wenig Neigung zu Vor-oder Rückschritten; daher stammt die alte klinische Angabe, daß Asthma und Tuberkulose sich gegenseitig meist ausschließen. Man darf sich darauf aber durchaus nicht verlassen, und ich habe genug traurigste Beispicle dafür erlebt, daß über den augenfälligen Überempfindlichkeiten anderer Art eine fortschreitende Tuberkulose übersehen wurde und dann nach langen Jahren des Unbemerktbleibens unaufhaltsam zum Tod geführt hat.

In dieser Hinsicht sind also auch die sonst so außerordentlich zutreffenden und genauen Angaben Hollos zu vervollständigen. Ich glaube ihm sehr gerne, daß er unter seinem Sanatoriumsmaterial aus derartigen ganz leichten, überempfindlichen, generalisierten sekundären Tuberkulosen der Lunge, die er im Anschluß an meine Untersuchungen als ,juvenile Form“ der Lungentuberkulose der Phthise gegenüberstellt, noch keine schwere Lungentuberkulose hat entstehen sehen. Es ist das erstens selten und braucht zweitens sehr lange Zeit, aber unmöglich ist es nicht. Je mehr er sein Augenmerk auch auf die schwereren derartigen Formen richten wird und die Anamnese der destruktiven Tuber- 
kulosen rückverfolgt, desto eher wird er gelegentlich auch gleiche Beobachtungen machen können.

Je leichter diese generalisierenden Tuberkulosen sind, um so mehr tritt naturgemäß der tuberkulöse Herd in den Hintergrund, so daß schließlich die geschilderten Überempfindlichkeiten als einziges bemerkbares Krankheitssymptom übrig bleiben. Man darf natürlich nicht in den Fehler verfallen, alle solchen Überempfindlichkeiten der Tuberkulose zur Last zu legen. Es kommen hier offenbar dispositionelle Momente, vielleicht auch Virulenzgrade des Erregers mit ins Spiel, selbst soweit es sich um nur durch den Kontakt mit dem tuberkulösen Virus ausgelöste Überempfindlichkeiten handelt. Es gibt aber, namentlich im Kindesalter, eine große Reihe andersartig ausgelöster Überempfindlichkeiten, die bei der eigenartigen Stereotypie dieser Reaktionsform den tuberkulösen ungemein ähnlich sehen können. Die Entscheidung bringt der Versuch mit dem Nachweis der Tuberkulinempfindlichkeit, wobei unter Tuberkulin alle Derivate des Tuberkelbacillus zu verstehen sind.

Die leichtesten Formen der hierhergehörigen Lungenerkrankungen sind chronische Bronchitiden von gewöhnlich eigenartiger Lokalisation, die schon dadurch den Gedanken an ihr Ausgelöstsein durch lokale Krankheitsherde sehr nahelegen. Doch bilden sich auch ganz allgemeine chronische Bronchitiden aus, wie ja auch die Asthmafälle, die hierher gehören, die ganzen Lungen ergreifen. Je größer nun die lokalen tuberkulösen Herde werden, um so mehr beteiligen sie sich am Krankheitsbild. Es ist selbstverständlich, daß nur ganz leichte Tuberkulosen längere Zeit ohne Ausbildung größerer Lokalherde verlaufen können, daß auch im übrigen die Größe des Lokalherdes ein sehr wesentliches Charakteristikum der Schwere der Erkrankungen darstellt. (Turban!) Bei dem rekurrierenden Charakter der Tuberkulose gilt dieser Satz nicht unmittelbar für das Augenblicksbild; wohl aber muß jede Tuberkulose, die einmal zur Ausbildung von großen Herden geführt hat, zu einer Zeit ihrer Entwicklung eine schwere Erkrankung gewesen sein. Die rekurrierende Natur der Tuberkulose, ganz besonders aber der Lungentuberkulose, macht allen diesen einmal schwer gewesenen Formen gegenüber auch für die weitere Prognose große Rerserve zur Pflicht. Es ist selbstverständlich, daß für diese Beurteilung nur der eigentliche tuberkulöse Herd und nicht die wechselnde Ausdehnung der Randreaktion in Betracht kommt. Sekundäre Lungentuberkulosen, bei denen die eigentlichen tuberkulösen Herde in den Vordergrund der klinischen Eischeinungen treten, sind demnach ganz anders zu beurteilen, als die chronischen Bronchitiden, bei denen nur die entzündliche Reaktion der näheren und ferneren Umgebung klinische Erscheinungen macht. Sowie einmal größere Dauerherde da sínd, so stellt sich auch die intrabronchiale Ausbreitung mit allen ihren Gefahren ein. Es sind zwar auch dann noch Heilungen möglich, aber sie sind sehr viel seltener und meist auch unvollständiger.

Auch die Vorgänge bei der Heilung ändern sich mit dem Eintritt der stärkeren Úberempfindlichkeit. Der isolierte Primärkomplex hat als eigene, für ihn 
ganz besonders typische Heilungsform die Verkalkung. Das akut entzündliche Reaktionsprodukt der frühen sekundären Zeit wird dagegen selbstverständlich einfach resorbiert. Ich habe durchaus den Eindruck, daß dabei auch das nekrotische oder nekrobiotische Zentrum des Herdes sich anders verhält als früher und später. Jedenfalls bleibt die typische schnelle Verkalkung aus, und ebenso sicher ist es, daß auch nekrotische Partien verflüssigt und ausgestoßen werden. Wieviel dabei von dem verflüssigten Käse auch resorbiert werden kann, ist noch eine Streitfrage. Meine Beobachtungen sprechen auch für weitgehende Resorption, und Eugen Albrecht hat ja schon vor vielen Jahren darauf hingewiesen, daß wir nicht wissen, was aus den großen käsigen Drüsen wird in allen denjenigen Fällen, die nicht in diesem Stadium zum Absterben kommen. Ihm war dabei auffällig, daß man bisher keine mit Sicherheit für sie in Anspruch nehmbare Residuen aufzufinden vermochte. Das würde also mit der Annahme einer Resorption zum mindesten nicht in Widerspruch stehen, solange man nicht annehmen will, daß sie alle ausnahms. los noch im Stadium der großen Drüsentumoren zum Tode führen, was wir für die sichtbaren Drüsengr uppen mit vollster Sicherheit ausschließen können. Das eine ist jedenfalls sicher, $d a \beta$ bei den akuten entzündlichen Randreaktionen ein geradezu ungeheueres Lymphocytenmaterial in den Herd gelangt und in ihm zugrunde geht. Daß dabei verdauende und verflüssigende Enzyme frei werden, wissen wir ja auch aus anderen Beobachtungen. Wir können also diese Reaktionsweise geradezu als einen Versuch bezeichnen, sich des Herdes durch Verflüssigung und Resorption oder Ausstoßung zu entledigen.

Diese Neigung der sekundären Herde zur Einscnmeizung iührt in der Lunge naturgemäß zur Kavernenbildung. Diese Kavernen werden lange nicht so rasch und regelmäßig in schwielige Massen eingebettet als bei der tertiären Lungentuberkulose. Sie können deshalb sowohl ungeheuer rasch fortschreiten, wie aber auch nach Ausstoßung ganz erstaunlich rasch und vollständig schrumpfen. Jeder, der über ein größeres Kindermaterial verfügt, weiß, daß bei ihnen gelegentlich Kavernen in anderer Weise, und zwar viel schneller und vollständiger bis zum vollkommenen Verschwinden ausheilen, und der Grund für diese Möglichkeit liegt eben in der andersartigen Reaktion der Herdumgebung. Es ist also offenbar ähnlich wie bei der Verkalkung des isolierten Primärkomplexes, die eben auch mit der Allergie dieses Stadiums offenbar schon unmittelbar mitgegeben ist.

Die schweren sekundären Lungentuberkulosen unterscheiden sich von den echten tertiären Formen durch ihre Zugehörigkeit zu einer generalisierten Tuberkulose des Gesamtorganismus.

Diese Zugehörigkeit verrät sich sowohl in der Anordnung der Krankheits. herde, also vor allem dem Miterkranken der Drüsen und der Lunge selbst, dann aber auch durch das Vorhandensein tuberkulöser Herde auch im Gebiet des großen Kreislaufes. Wo größere sichtbare Weichteil- and Knochen herde vorhanden sind oder hämatogene Erkrankungen innerer Organe, z. B. des Genitaltraktus, sich nachweisen lassen, ist die Erkennung ungeheuer einfach. Besonders wichtig für die Unterscheidung ist die genaue. Untersuchung des gesamten Lymphdrüsensystems. Wo sich eine aktive Miterkrankung sekundären Typs, $-d$. r. also mit den anfallsweisen entzündlichen Attacken und unter Ausbildung größerer Drüsentumoren - z. B. an den Hals- oder Achseldrüsen nachweisen läßt, ist der 
sekundäre Charakter der Erkrankung sichergestellt. Wo das nicht gelingt, ist die Unterscheidung schon sehr viel schwieriger. Man ist dann auf die Symptome von seiten der Lungenerkrankungen allein angewiesen. und wird auch hier vor allem nach der Mitbeteiligung der Drüsen fahnden. Die typischen entzündlichen Realstionen sind auch in der Lunge für die Diagnose dann der wichtigste Zug des Krankheitsbildes.

\section{Isolierte Phthise}

Uber das Krankheitsbild der isolierten Phthise brauche ich in Ihrem Kreise nicht viel Worte zu machen. Es ist ganz allgemein wesentlich bekannter als das der sekundären Formen. Für das klinische Bild steht das Fehlen hämatogener Herde im Gebiet des großen Kreislaufs, das vollkommene Zurücktreten aktiver Drüsenherde und eine ganz eigenartige Isolierung des Herdes auch noch innerhalb der Lunge im Vordergrund. Eine echte Phthise, die hervorstechende rein bronchitische Züge zeigt, ist immer eine Komplikation oder eine Fehldiagnose. So häufig die exsudativ-bronchitischen Reaktionen in der sekundären Zeit sind, um so mehr treten sie zurück, je weiter dic Entwicklung der Krankheit fortschreitet. Im klinischen Bild dominiert nun der tuberkulöse Herd selbst. Die Reizantwort hat sich noch einmal geändert und zwar durchaus in der Richtung auf eine gewisse Gewöhnung oder Immunität. Die Beobachtung weist also auch hier in der gleichen Richtung wie bei der Beachtung der Anordnung der Krankheitsherde. Wenn die humoralen Metastasen schließlich ganz ausbleiben, haben wir auch eine andere Herderkrankungsform in der Lunge. Kleine oder größere broncho-pneumonische oder acinöse Herde können direkt neben rein vesiculär atmendem, normal gebautem und funktionierendem Lungengewebe liegen ohne jede entzündliche Irritation. Wohl kommen noch, exsudative" Formen auch in diesem Stadium vor; sie sind aber dann durch rasches Wachstum der Herde oder durch schwere alterierende Giftwirkung an neuen Organstellen verursacht. Sie sind nicht mehr vorwiegend aktiv, sondern passiv, nicht mehr vorwiegend Entzündung, sondern vorwiegend Nekrobiose, um im alten Virchowschen Bilde zu bleiben.

Es ist selbstverständlich, daß die Entzündung nur zurücktritt, nicht vollkommen fehlt und daß sie mehr den prolferierenden Charakter der chronischen Entzündung annimmt als den exsudativen der akuten. Mit dem Fehlen der stürmischen Randreaktionen wird das ganze Geschehen im Krankheitsherde träger und torpider, soweit nicht besonders kräftige Aktionen von seiten des Krankheitserregers auftreten. Die typische „Heilung" ist eine ungemein chronische und reichliche Narbenbildung, die sich über viele Jahre wegziehen kann, ohne zu einer eigentlichen Vernarbung zu führen, weil die nekrotischen Herdteile nicht beseitigt werden können. Man sieht jetzt nie mehr die weitgehende klinische Restitutio ad integrum, die bei den akuten reaktiven Entzündungen der sekundären Zeit und auch noch in den Herdheilungen dieser Entwicklungsphase so auffällig ist. Die Heilung erfordert ungeheuer viel Zeit, ungeheuer viel Geduld und Konsequenz und eine Sorgfalt, deren nur ein vergleichsweiser geringer Prozentsatz von Patienten und Ärzten sich fähig zeigt. Wer rasche Erfolge sehen will, und wer in derartiger geduldiger Kleinarbeit seine Befriedigung nicht finden kann, der darf nicht Sanatoriumsarzt werden. 
Ich habe schon davon gesprochen, daß bei diesen tertiären Tuberkulosen neben dem Zurücktreten der Überempfindlichkeitsreaktion als aktiver Reizantwort von seiten des Organismus sich doch häufig sehr schwere schädigende Viruswirkungen bemerkbar nachen. Ich möchte zum Schluß noch darauf aufmerksam machen, daß auch diese Erscheinungen, so sonderbar das klingen mag, einen eigenartigen Zusammenhang mit der Immunitäl dieser Stadien besitzen. Sie wissen, daß außer der echten Anaphylaxie, deren typischstes Beispiel die Sensibilisierung durch Vorbehandlung mittels parenteraler Einverleibung irgendeiner Eiweißart darstellt, noch ein zweites Phänomen vorkommt, das mit ihr auf den ersten Blick verwechselt werden kann. Es ist das die paradoxe Reaktion Behrings. Dabei handelt es sich um ein bisher unerklärtes Wiedergiftigwerden eines bestimmten Toxins für ein gegen dasselbe hoch immunisiertes Tier. Die Erscheinung ist von Behring an gegen Diphtherie und Tetanus immunisierten Tieren beobachtet worden. Es kommt gelegentlich vor, daß ein sagen wir z. B. gegen Tetanus hochimmunisiertes Tier, von dessen Serum wenige Kubikzentimeter ein anderes Tier gegen die Wirkung der mehrfach tödlichen Dosis dieses Giftes mit Sicherheit zu schützen vermag, trotzdem selbst so empfindlich gegen dieses Gift ist, daß es schon bei winzigem Bruchteil der tödlichen Dosis und zwar nicht unter anaphylaltischem Schock, sondern unter Erscheinungen der Tetanuswirkung zugrunde geht. Hier handelt es sich offenbar um etwas grundsätzlich von der typischen Reizantwort der Anaphylaxie Verschiedenes. Die spezifische Giftempfindlichkeit ist hier auffallend gesteigert.

Sehen wir uns nun das Krankheitsbild der echten isolierten Phthise näner an, so finden wir neben den geschilderten Zügen einer deutlichen Immunität auch ganz ungemein ausgesprochen Züge einer solchen hohen spezifischen Giftempfindlichkeit. Hierher gehören die sog. tuberkulo-toxischen Wirkungen auf das Herz, auf die Haut, auf das Nervensystem und auf die Gesamternährung, also die Tachykardie und Labilität des Pulses, die eigentümliche trockene abschilfernde Haut, die Nachtschweiße und die allgemeine Kachexie des Phthisikers. Es ist mir immer schon von größter Wichtigkeit gewesen, daß damit auch eine Änderung der Reaktionsweise auf das Tuberkulin Hand in Hand geht. Auch bei den Tuberkulininjektionen tritt bei den sekundären Formen die stereotyp entzündliche, also sagen wir einmal die anaphylaktische Reaktion, besonders stark hervor, während die Allgemeinreaktion stark zurücktritt. Wir können hier ganz enorme Stichreaktionen und auch noch starke Herdreaktionen sehen ohne Temperatursteigerung und bei nur ganz geringem allgemeinem Krankheitsgefühl. Umgekehrt bei der Phthise, und zwar hier wieder gerade bei den Formen, die die spezifischen Giftwirkungen schon spontan deutlich zeigen. Hier können winzige Tuberkulindosen, Millionstel eines Milligramms, schwerstes allgemeines Unbehagen, Temperatursteigerung, Vermehrung der Nachtschweiße hervorrufen, ohne daß auch nur die geringste Stichraktion dabei wahrzunehmen ist. Es ist selbstverständlich, daß dieser Unterschied am deutlichsten in der Nähe der Reizschwelle hervortritt, also an der jeweiligen Reaktionsgrenze der Fälle. Bei höheren Dosen mischen sich auch die anderen Reaktionsformen der vorherrschenden bei und erschweren damit die Beurteilung.

Die spezifische Giftüberempfindlichkeit des immunen Tieres gewinnt damit 
ein sehr großes praktisches Interesse, denn was ich hier vorgetragen habe, sind keine Phantasien, sondern Beobachtungen. Es wird deshalb wahrscheinlich; daß wir aus dem Studium der spezifischen Giftüberempfindlichkeit Handhaben für die Behandlung der isolierten Phthise gewinnen werden, wenn wir einmal wisssen werden, wodurch sie hervorgerufen und womit sie wieder beseitigt werden kann. Dann werden wir dieser ungemein komplizierten Erkrankung mit ganz anderen Verständnis- und Behandlungsmöglichkeiten gegenüberstehen. Das eine ergibt sich ja schon ohne weiteres, daß bei derartigen Differenzen in der Tuberkulinwirkung von einer wissenschaftlichen Tuberkulinbehandlung erst gesprochen werden kann, wenn wir die Erkrankung selbst genauer kennen. Es geht schon heute nicht mehr an, Tuberkulintherapie zu treiben, ohne die biologisch sich so deutlich unterscheidenden Entwicklungsformen der Tuberkulose zu berücksichtigen. Gerade die Gefahr der „Giftüberlastung“, besonders der tertiären Formen, ist ja von allem Anfang an von den Praktikern erkannt worden. Der Umschlag aus einer die Heilung herbeiführenden Immunität in die spezifische Giftüberempfindlichkeit des Immunen kann zweifellos bei der Tuberkulose auch durch Tuberkulinbehandlung erzeugt werden. Das Tuberkulin löst jeweils auch in seiner künstlichen Anwendung nur die spontanen Reaktionen der Entwicklungsstufen aus, in der die Erkrankung gerade steht. Tuberkulintherapie und Tuberkulintherapie können also zwei durchaus verschiedene Dinge sein. Es handelt sich bei ihr nicht um die Einverleibung eines Schutzstoffes, sondern um künstliche Krankheit. Wer sich darüber klar geworden ist, welche ungeheuren Verschiedenheiten innerhalb der menschlichen Tuberkulose sich finden, der wird sich nicht mehr darüber wundern, daß das künstliche Eingreifen mit dem gleichen Virus, das in seiner spontanen Entwicklung so unberechenbar ist, nicht auf ein einfaches Schema gebracht werden kann. Es darf aber nicht vergessen werden, daß gerade die Entdeckung des Tuberkulins uns für die Erforschung der menschlichen Tuberkulose ganz unschätzbare Dienste geleistet hat. Die richtige therapeutische Ausnutzung ist nur mehr eine Frage der Zeit.

\section{Dr. Kurt Nicol (Koburg):}

\section{Die pathologisch-anatomischen Grundlagen der Lungenphthise und ihre Bedeutung fuir die klinische Einteilung der Verlaufsformen.}

M. H.! Als ich im September 1913 auf Ihrer Tagung in Freiburg über eine neue Nomenklatur und Einteilung der Lungenphthise auf Grund neuer pathologisch-anatomischer Anschauungen sprach, war ich mir wohl bewußt, daß ich damit einen. Stein ins Rollen brachte, der längere Zeit braucht, bis er wieder zur Ruhe kommt. Die Notwendigkeit und das allgemeine Verlangen nach einer auch. den Praktiker befriedigenden Nomenklatur und Einteilung der Lungenphthise ist in der damaligen Diskussion allseitig deutlich zum Ausdruck gebracht worden, und eine größere Zahl Thres Kreises, Brauer, Liebe, Wolf u. a. griffen die Vor-, schläge freudig auf. Für manche waren sie natürlich ein Neuland, das sie nur. vorsichtig betreten wollten. Liebe war es, der damals aussprach, die Weiterdurcharbeitung der von Aschoff und mir gemachten Vorschläge würden aus dem. allgemein unbefriedigenden und nebelhaften Zustande herausführen: Ja, es war 